\title{
Airway Findings of Nasolaryngoscopy and Cephalometry Associated with Obstructive Sleep Apnea
}

\author{
Jorge Espinoza, DDS ${ }^{1}$; Eliana Reyes, DDS ${ }^{1}$; Patricia Hidalgo, MD, MS²; Juan Ospina, MD³; Liliana Otero, MD, MS ${ }^{4}$ \\ ${ }^{1}$ Pontificia Universidad Javeriana, Colombia; ${ }^{2}$ Hospital Universitario San Ignacio, Pontificia Universidad Javeriana, Colombia; ${ }^{3}$ Department \\ of Otolaryngology, Hospital Universitario San Ignacio, Pontificia Universidad Javeriana, Colombia; ${ }^{4}$ Centre of Dental Research, Pontificia \\ Universidad Javeriana, Colombia
}

\begin{abstract}
Study Овjectives: Obstructive sleep apnea (OSA) is associated with obstruction of the upper airway. The objectives of the current study were to determine the correlation between the findings of the fiberoptic nasolaryngoscopy and lateral cephalometry, and to evaluate the relationship between obstructions in the airway identified in both diagnostic methods and OSA in adults.

Methods: Fiberoptic nasolaryngoscopy and lateral cephalometry were performed in 29 patients with OSA (7 men and 22 women) and 24 healthy subjects (9 men and 15 women). Obstructions in nasopharynx, hypopharynx, and larynx areas were identified with nasolaryngoscopy. Cephalometric measures included upper and lower pharynx spaces, hyoid bone position, mandibular and maxilla size and position, and skeletal malocclusion. Concordance between the findings reported in both diagnostic tools were evaluated by kappa test and $\chi^{2}$, student $t$ and Mann-Whitney $U$ tests were used for association analysis and adjusted by age, sex, and weight.

RESULTS: No correlation between cephalometric and nasolaryngoscopy measurements were found. Statistical significant difference was shown for obstructions in hypopharynx and OSA (odds ratio 4.06, confidence interval 1.10-14.89, $P=.034$ ). The superior position of hyoid bone was found as a protective factor for OSA (odds ratio 0.02, confidence interval 0.001-0.57, $P=.02$ ).
\end{abstract}

Conclusions: This study showed no correlation between fiberoptic nasolaryngoscopy and cephalometric measures. Hypopharynx obstructions identified by nasolaryngoscopy were associated with OSA in adults.

KEYWORDS: airway, cephalometry, nasolaryngoscopy, obstructive sleep apnea

Citation: Espinoza J, Reyes E, Hidalgo P, Ospina J, Otero L. Airway findings of nasolaryngoscopy and cephalometry associated with obstructive sleep apnea. Journal of Dental Sleep Medicine. 2018;5(1):5-10.

\section{INTRODUCTION}

Obstructive sleep apnea (OSA) is a sleep disorder characterized by cessation of partial airflow (hypoapnea) or complete obstruction (apnea) of the upper airway for at least 10 seconds. ${ }^{1,2}$ Although most people with OSA remain undiagnosed, ${ }^{3}$ the estimated prevalence of OSA is $9 \%$ of adult women and $24 \%$ of adult men. ${ }^{4}$ In Latin America, the prevalence of OSA in adults is approximately $4.6 \%$ in men and $2.7 \%$ in women. ${ }^{5,6} \mathrm{In}$ Colombia, the prevalence of sleep disorders has been reported at $26.9 \%$ according to the STOP-BANG questionnaire.?

The diagnostic test for OSA is polysomnography, and it is considered the gold standard. ${ }^{8}$ Polysomnography cannot show the sites of obstruction in the airways. Diagnostic tools that can show airway obstructions include computed tomography, magnetic resonance imaging, lateral radiography with cephalometry, and fiberoptic nasolaryngoscopy. ${ }^{9-11}$ Cephalometry and fiberoptic nasolaryngoscopy are diagnostic aids that help to identify the exact area of the obstruction, which is important for determining treatment and prognosis in each individual with OSA. ${ }^{12}$

Fiberoptic nasolaryngoscopy is a simple and routine examination, performed mostly by the otolaryngologist, in order to identify the area of collapse in patients with OSA in threedimensional assessment of airway. ${ }^{13-15}$ This collapse can occur at different levels such as the nasopharynx, hypopharynx, and larynx. The hypertrophy of palatine adenoids and tonsils, and alterations in the morphology of the retropalatine area, are the anatomic areas most associated with the presence and severity of OSA. ${ }^{16}$

Lateral cephalometry is another diagnostic tool used for identifying airway obstructions associated with OSA. Cephalometric measures that have shown greater association with the diagnosis of OSA are: mandibular retrognathism, mandibular micrognathism, increased ANB angle, lower position of the hyoid bone, and decrease in upper and lower pharynx space, ${ }^{13,17}$ but craniofacial features associated with OSA are diverse in different populations and ethnic groups. ${ }^{18,19}$

Identification of obstruction areas using cephalometry in conjunction with other methods such as endoscopic studies in patients with OSA is important for diagnosis, prognosis, and treatment. ${ }^{20-22}$ Some obstructions associated with OSA should be treated with surgery or oral devices, and for that reason, determining the concordance between fiberoptic nasolaryngoscopy and cephalometry is necessary because these two tests allow the spatial assessment of both skeletal and soft tissue structures. Barbosa et al..$^{23}$ and Souki et al. ${ }^{24}$ showed a high degree of correlation between the cephalometric measurements and nasopharynx space measured by fiberoptic nasolaryngoscopy in children, but there are few studies about this correlation in adults. ${ }^{13}$

The objectives of the current study were to determine the correlation between the findings of the fiberoptic nasolaryngoscopy and lateral radiographies, and to evaluate the 
Figure 1-The cephalometric variables analyzed.

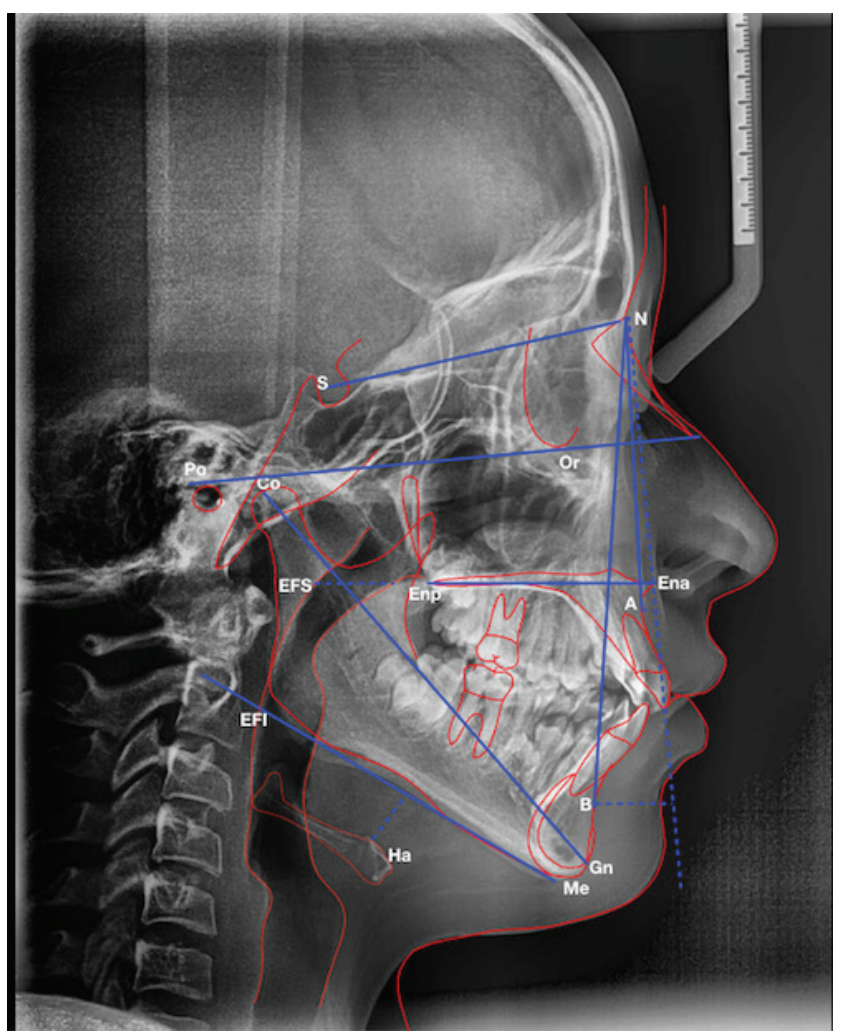

SNA = angle formed for plane sella nasion, from sella (at the center of pituitary fossa of sphenoid bone) to nasion (most anterior point on frontonasal suture) and nasion $\mathrm{A}$ (most concave point of anterior maxilla) plane. SNB = angle from sellanasion plane and nasion-B (most concave point on mandibular symphysis). ANB = angle $A$ point-nasion- $B$ point. Position of the hyoid bone = distance the hyoid bone was measured perpendicularly to mandibular plane. Mandibular length Co$\mathrm{Gn}=$ plane from $\mathrm{Co}$, most posterior superior point of the condyle of mandible to $\mathrm{Gn}$, midpoint between pogonion and menton. ENA-ENP = anterior point of maxilla to posterior limit of maxilla or palate. Maxilla position $\mathbf{N} \perp \mathbf{A}=$ nasion perpendicular $\mathrm{A}$. Mandible position $\mathrm{Pg} \perp \mathbf{N}=\mathrm{Pg}$ to nasion perpendicular. Upper pharynx space $=$ point on the posterior outline of the soft palate to the closest point on the posterior pharyngeal wall. Lower pharynx space $=$ intersection of the posterior border of tongue and the inferior border of the mandible to the closest point on the posterior pharyngeal.

relationship between obstructions in the airway identified in both diagnostic methods and obstructive sleep apnea in adults.

\section{METHODS}

\section{Sample and Design}

A convenience sample included 53 adults aged between 18 and 72 years from Sleep Clinic at Hospital Universitario San Ignacio with symptoms suggestive of sleep apnea. The study was approved by the ethics committees of the Faculty of Medicine at the Pontificia Universidad Javeriana and Hospital Universitario San Ignacio. After polysomnography, 7 men and 22 women with OSA, and 9 healthy men and 15 healthy women (without OSA) were selected. Informed consent was obtained from all subjects participating. Individuals with craniofacial anomalies, syndromes, cleft lip and palate; patients with neurological and/or neuromuscular alterations; patients who have undergone orthognathic surgery or surgical intervention of adenoids; and patients with central apnea were excluded.

This study included two study designs: (1) correlation between fiberoptic nasolaryngoscopy and lateral cephalometry findings in healthy subjects and patients with OSA and (2) crosssectional association between OSA and obstructions identified in fiberoptic nasolaryngoscopy and lateral cephalometry.

\section{Airway Obstructions Identification}

Fiberoptic nasolaryngoscopy was performed in supine and seated positions for each patient previously specified by the Otorhinolaryngology Service of Hospital Universitario San Ignacio. Obstructions in nasopharynx (septum and turbinate), hypopharynx (uvula, palatine and lingual tonsils), and larynx (epiglottis, ventricular bands, and piriform sinuses) areas were identified.

Skull lateral radiographs were obtained by the same operator in the same equipment. This examination was performed in the natural head position and at the end of expiration to standardize hyoid bone position. Cephalometric measures were used to evaluate the craniofacial and airway phenotype in each subject. Cephalometric measures included upper and lower pharynx spaces, hyoid bone position, mandibular and maxilla size and position, and skeletal malocclusion (Figure 1). Cephalometric analysis was performed by two researchers using a $0.5-\mathrm{mm}$ caliper pencil and a standard cephalostat. Intraobserver and interobserver agreement was obtained for cephalometric analysis. The otorhinolaryngologist and orthodontist were blinded to the results of polysomnography of each patient.

\section{Correlation Study}

For the correlation study, the following variables of cephalometry and the fiberoptic nasolaryngoscopy were correlated:

1. Decreased upper pharynx space (cephalometry) with nasopharyngeal obstruction (septum and turbinate identified by nasolaryngoscopy).

2. Decreased lower pharynx space (cephalometry) with obstruction in the hypopharynx (uvula, palatine and lingual tonsils identified by nasolaryngoscopy).

3. Inferior position of hyoid bone (cephalometry) with laryngeal obstruction (epiglottis, ventricular bands and piriform sinuses identified by nasolaryngoscopy).

\section{Cross-Sectional Association Study}

This study was performed to evaluate the relationship between cephalometric and fiberoptic nasolaryngoscopy findings and OSA, comparing findings from healthy subjects and subjects with OSA. Cephalometric measurements are shown in Figure 1. Structures evaluated in nasolaryngoscopy included septum, turbinate, uvula, palatine and lingual tonsils, epiglottis, ventricular bands, and piriform sinuses.

\section{Statistical Analysis}

For the correlation study, a kappa test was performed between cephalometric and fiberoptic nasolaryngoscopy variables. For 
Table 1-Distribution of sample by age, sex, and BMI.

Feature
Age, years, median (IQR)
Sex, n (\%)
Male
Female
BMI, n (\%)
Normal
Low weight
Overweight

OSA $(\mathbf{n}=29)$
$42(31-48)$
$7(24.1)$
$22(75.9)$
$19(65.5)$
$8(27.6)$
$2(6.9)$

Healthy $(\mathbf{n}=\mathbf{2 4})$
$54(42.5-61)$
$9(37.5)$
$15(62.5)$
$12(50)$
$5(20.8)$
$7(29.2)$

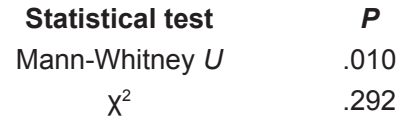

Fisher exact

.117

$\mathrm{BMI}=$ body mass index, IQR = interquartile range, OSA = obstructive sleep apnea .

Table 2-Correlation analysis for cephalometry and fiberoptic nasolaryngoscopy.

Feature

Upper pharynx space versus Nasopharyngeal obstruction

Lower pharynx space versus Obstruction in hypopharynx

Position of hyoid bone versus Laryngeal obstruction

$\begin{array}{cc}\text { Agreement } & \begin{array}{c}\text { Estimate } \\ \text { Agreement }\end{array} \\ 14 \% & 12 \% \\ 63 \% & 62 \% \\ 0 \% & 0 \% \\ & \\ & \\ & \\ & \\ & \\ & \end{array}$

cross-sectional association study, $\chi^{2}$ or Fisher exact tests were $t$ tests were used for quantitative variables in according to the normal distribution of variables previously established with the Kolmogorov-Smirnov test.

Bivariate analysis was performed to evaluate the relationship between cephalometric measurements and findings of the fiberoptic nasolaryngoscopy with OSA. Odds ratio (OR) and $P$ value with $95 \%$ confidence interval (CI) were calculated. As proof of goodness of fit, the pseudo $R^{2}$ was used (a test analogous to $R^{2}$ that indicates the goodness of fit of the model to the data). Although it does not have the immediacy of $R^{2}$ 's interpretation of linear regression that directly indicates which proportion of the variance of the dependent variable is explained by the model, it is an approximation based on a comparison of the likelihood of the model only with the constant. The statistical software used for the analysis was STATA version 13.0 (StataCorp LLC, College Station, Texas, United States).

\section{RESULTS}

A total of 53 adults were included in the study, with median age of 42 years for healthy subjects and 54 years for subjects with OSA. The distribution for sex was similar between healthy subjects and those with OSA, with a higher proportion of women $75 \%(n=29)$ in the healthy group and $62 \%(n=24)$ in the OSA group. The body mass index was similar between both groups (Table 1).

Results for intraobserver and interobserver agreement for cephalometric analysis showed $98.1 \%$ corresponding to kappa $=0.97$, indicating an excellent correlation between the observers. There was no correlation between the variables evaluated in the fiberoptic nasolaryngoscopy and cephalometry (Table 2). Obstructions in the hypopharynx (uvula, palatine and lingual tonsils) showed statistical significant association with OSA (OR 4.06, CI 1.10-14.89, $P=.034$ ), Table 3. There were no statistical significant differences between supine and seated positions (Table 4). In cephalometric analysis, it was observed that ascending position of hyoid bone was found as a protective factor for OSA (OR 0.02, CI 0.001-0.57, $P=.02$ ), Table 5.

\section{DISCUSSION}

The concordance analysis of this investigation did not reveal any correlation between the evaluated features in fiberoptic nasolaryngoscopy and cephalometry. In a similar study, Narayanan and Faizal ${ }^{13}$ evaluated 70 patients with OSA and demonstrated correlation between retropalatal collapse and retroglossal region observed in nasopharyngolaryngoscopy with distance from mandibular plane to hyoid bone measured in lateral cephalometry, but Koo et al. ${ }^{25}$ showed no correlation between retropalatal region and mandibular plane to hyoid bone. However, these studies included patients with OSA, and in our study, we compared structures in subjects with and without sleep apnea.

Concordance in the obstruction of retropalatal and retroglossal regions have been demonstrated in individuals with OSA through fiberoptic nasolaryngoscopy and magnetic resonance imaging, but it seems that the obstruction is associated with the severity of apnea-hypopnea index. ${ }^{26}$ Future studies should be performed to investigate the concordance between lateral cephalometry and fiberoptic nasolaryngoscopy in patients with moderate and severe OSA. Additionally, although positive correlation between lateral cephalometry and nasolaryngoscopy has been reported for studies of OSA in children, nasolaryngoscopy is superior to cephalometry in assessing obstructions in nasopharynx and hypopharynx 
Table 3-Fiberoptic nasolaryngoscopy findings.

Feature

Septal deviation

No

Yes

Cornet hypertrophy

No

Yes

Adenoid hypertrophy

No

Yes

Hypertrophy of palatine tonsils

No

Yes

Obstruction of the nasopharynx

No

Yes

Obstruction of the hypopharynx

No

Yes

Obstruction of the larynx

No

Yes
Comparison Between Healthy and OSA Groups OR $(95 \% \mathrm{Cl})$

$P$

1

$0.60(0.14-2.57)$

.499

1

$1.45(0.49-4.31)$

.499

1

$2.22(0.72-6.82)$

1

$0.53(0.09-3.40)$

.536

1

$2.3(0.67-7.79)$

.181

1

$4.06(1.10-14.89)$

.034

1

$3.99(0.38-41.22)$

.244

$\mathrm{Cl}=$ confidence interval, $\mathrm{OR}=$ odds ratio, OSA = obstructive sleep apnea.

Table 4-Fiberoptic nasolaryngoscopy findings (seated and supine).

$\begin{array}{lrr}\begin{array}{l}\text { Feature } \\ \text { Obstruction of the nasopharynx seated }\end{array} & \text { OSA ( } \mathbf{n}=\mathbf{2 9}) & \text { Healthy }(\mathbf{n}=\mathbf{2 4}) \\ \text { No } & 23(79.3) & 15(62.5) \\ \text { Yes } & 6(20.7) & 9(37.5) \\ \text { Obstruction of the hypopharynx seated } & 13(44.8) & 4(16.7) \\ \quad \text { No } & 16(55.2) & 20(83.3) \\ \text { Yes } & & \\ \text { Obstruction of the larynx seated } & 28(96.6) & 21(87.5) \\ \text { No } & 1(3.4) & 3(12.5) \\ \text { Yes } & 23(79.3) & 14(58.3) \\ \text { Obstruction of the nasopharynx supine } & 6(20.7) & 10(41.7) \\ \text { No } & 18(62.1) & 10(41.7) \\ \text { Yes } & 11(37.9) & 14(58.3) \\ \text { Obstruction of the hypopharynx supine } & & \\ \text { No } & 28(96.6) & 20(83.3) \\ \text { Yes } & 1(3.4) & 4(17.7) \\ \text { Obstruction of the larynx supine } & \end{array}$

Values are presented as $n(\%)$. OSA = obstructive sleep apnea.

because cephalometry tends to underestimate the tonsillar hyperplasia compared with nasopharyngoscopy. ${ }^{27}$

Our results showed statistically significant association between obstructions at the hypopharynx through fiberoptic nasolaryngoscopy (increase size of uvula and palatine and lingual tonsils) and OSA $(P=.034, \mathrm{OR}=4.06,95 \% \mathrm{CI}=1.10-$ 14.89), but in lateral cephalometry these obstructions were not evident, although reduced lower pharynx space in patients with severe OSA has been reported. ${ }^{28}$ Reduced hypopharynx width has also been observed in adults with OSA due to the increase of velum and posterior pharyngeal wall thickness. This condition is usually observed in obese subjects, ${ }^{29}$ but in our study obese subjects were not included and reduced hypopharynx width was observed by nasolaryngoscopy in those who were overweight in the healthy group and the OSA group, but not by cephalometry. In children, adenotonsillar hypertrophy has shown to be the most common cause of pediatric OSA but OSA persists after adenotonsillectomy, suggesting that other factors such as craniofacial phenotype, obesity, and other comorbidities play an important role in the obstruction of retropalatal and 
Table 5-Cephalometric findings.

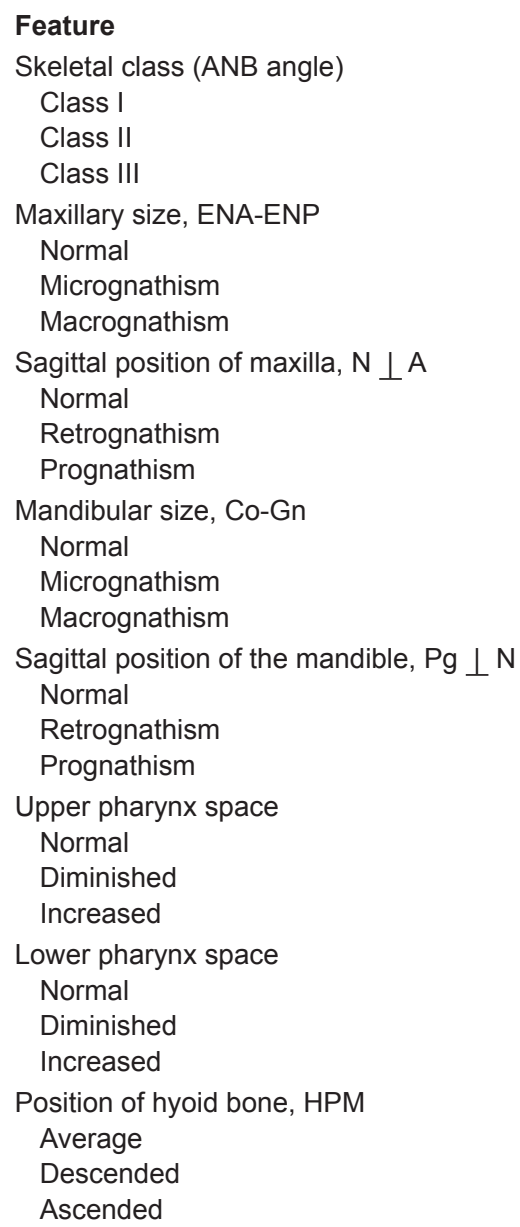

\begin{tabular}{|c|c|}
\hline Comparison Betwee & OSA \\
\hline OR $(95 \% \mathrm{Cl})$ & $P$ \\
\hline 1 & \\
\hline $0.75(0.10-5.23)$ & .772 \\
\hline NA & NA \\
\hline 1 & \\
\hline $1.14(0.12-10.38)$ & .906 \\
\hline NA & NA \\
\hline 1 & \\
\hline $2.66(0.15-45.14)$ & .497 \\
\hline $1.66(0.22-12.22)$ & .615 \\
\hline 1 & \\
\hline NA & NA \\
\hline NA & NA \\
\hline 1 & \\
\hline NA & NA \\
\hline $2.4(0.29-19.78)$ & .416 \\
\hline 1 & \\
\hline NA & NA \\
\hline $0.35(0.04-2.77)$ & .325 \\
\hline 1 & \\
\hline $4(0.32-48.65)$ & .277 \\
\hline NA & NA \\
\hline 1 & \\
\hline $0.8(0.03-17.19)$ & .887 \\
\hline $0.02(0.001-0.57)$ & .020 \\
\hline
\end{tabular}

ANB = angle A point-nasion-B point, $\mathrm{Cl}=$ confidence interval, $\mathrm{Co}-\mathrm{Gn}=$ plane from Co, most posterior superior point of the condyle of mandible to $\mathrm{Gn}$, midpoint between Pogonion and menton, ENA-ENP = anterior point of maxilla to posterior limit of maxilla or palate, HPM = hyoid to mandibular plane, $\mathrm{N} \perp \mathrm{A}=$ nasion perpendicular $\mathrm{A}, \mathrm{OR}=$ odds ratio, $\mathrm{OSA}=$ obstructive sleep apnea,

$\mathrm{Pg} \perp \mathrm{N}=\mathrm{Pg}$ perpendicular nasion.

retrolingual areas in children and in adults, although future studies are necessary to clarify the cause of obstruction in both regions associated with OSA. ${ }^{30}$ da Cunha Viana et al. ${ }^{31}$ evaluated patients age 18 to 65 years with moderate and severe OSA using fiberoptic nasolaryngoscopy under drug-induced sleep and demonstrated that retropalatine and retrolingual regions were the most frequent obstruction sites during induced sleep. In our study, we compared measurements in supine and seated positions in awake individuals by nasolaryngoscopy, but we did not find differences between measures in both positions; this fact could be explained because it seems that these structures are more affected by the sleep than by the position.

Findings in lateral cephalometry have shown strong evidence for reduced pharyngeal airway space, increased anterior facial height, and inferiorly placed hyoid bone in adults with OSA compared with controls. ${ }^{32}$ Inferiorly displaced hyoid with respect to the mandibular plane could be considered a risk factor for OSA and this position results from affected lingual musculature because of macroglossia, relaxation of the lingual musculature, or fat deposition. ${ }^{33}$ The outcomes of our study demonstrated that healthy patients have superiorly displaced hyoid bone suggesting that this condition could be a protective factor for OSA (OR 0.02, 95\% CI 0.001-0.57, $P=.02$ ).

This study showed that both cephalometry and fiberoptic nasolaryngoscopy are diagnostic tools that allow assessment of obstruction sites in adults with OSA. Future investigations should be directed toward comparison of the correlation between both tests in children and in patients with different severity of OSA and to identify the causes of obstructions in the upper airway associated with OSA.

\section{REFERENCES}

1. Lam B, Ooi CG, Peh WC, et al. Computed tomographic evaluation of the role of craniofacial and upper airway morphology in obstructive sleep apnea in Chinese. Respir Med. 2004;98(4):301-307.

2. Magliocca KR, Helman JI. Obstructive sleep apnea: diagnosis, medical management and dental implications. J Am Dent Assoc. 2005;136(8):1121-1129.

3. Kapur V, Strohl KP, Redline S, Iber C, O’Connor G, Nieto J. Underdiagnosis of sleep apnea syndrome in US communities. Sleep Breath. 2002; 6(2):49-54. 
4. Peppard P, Young T, Barnet J, Palta M, Hagen E, Hla K. Increased prevalence of sleep-disordered breathing in adults. Am J Epidemiol. 2013;177(9):1006-1014.

5. Yaggi HK, Strohl KP. Adult obstructive sleep apnea/hypopnea syndrome: definitions, risk factors, and pathogenesis. Clin Chest Med. 2010;31(2):179-186

6. Barros EL, Pradella-Hallinan M, Moreira GA, Stefanini Dde O, Tufik S, Fujita RR. Follow-up of obstructive sleep apnea in children. Braz J Otorhinolaryngol. 2014;80(4):277-284.

7. Ruiz AJ, Sepúlveda MA, Martínez PH, et al. Prevalence of sleep complaints in Colombia at different altitudes. Sleep Science. 2016;9(2):100-105.

8. Sutherland K, Lee RW, Cistulli PA. Obesity and craniofacial structure as risk factors for obstructive sleep apnoea: impact of ethnicity. Respirology. 2012;17(2):213-222.

9. Kuhle S, Urschitz MS, Eitner S, Poets CF. Interventions for obstructive sleep apnea in children: a systematic review. Sleep Med Rev. 2009;13(2):123-131.

10. Laxmi NV, Talla H, Meesala D, Soujanya S, Naomi N, Poosa M. Importance of cephalographs in diagnosis of patients with sleep apnea. Contemp Clin Dent. 2015;6(Suppl 1):S221-S226.

11. Kwentus J, Schulz SC, Fairman P, Isrow L. Sleep apnea: a review. Psychosomatics. 1985;26(9):713-724.

12. Georgalas C, Garas G, Hadjihannas E, Oostra A. Assessment of obstruction level and selection of patients for obstructive sleep apnoea surgery: an evidence-based approach. J Laryngol Otol. 2010;124(1):1-9.

13. Narayanan A, Faizal B. Correlation of lateral cephalogram and flexible laryngoscopy with sleep study in obstructive sleep apnea. Int $J$ Otolaryngol. 2015;2015:127842.

14. Aksoy EA, Serin GM, Polat S, Unal OF, Tanyeri H. The morphology of the nasopharyngeal inlet in obstructive sleep apnea. Eur Arch Otorhinolaryngol. 2014;271(4):771-775.

15. Wang JH, Chung YS, Cho YW, et al. Palatine tonsil size in obese, overweight, and normal-weight children with sleep-disordered breathing. Otolaryngol Head Neck Surg. 2010;142(4):516-519.

16. Williams RS, Lancaster JF, Karagama YF, Tandon SF, Karkanevatos A. A systematic approach to the nasendoscopic examination of the larynx and pharynx. Clin Otolaryngol Allied Sci. 2004;29(2):175-178.

17. Takai Y, Yamashiro Y, Satoh D, Isobe K, Sakamoto S, Homma S. Cephalometric assessment of craniofacial morphology in Japanese male patients with obstructive sleep apnea-hypopnea syndrome. Sleep Biol Rhythms. 2012;10(3):162-168.

18. Bharadwaj R, Ravikumar A, Krishnaswamy NR. Evaluation of craniofacial morphology in patients with obstructive sleep apnea using lateral cephalometry and dynamic MRI. Indian J Dent Res. 2011;22(6):739-748.

19. Vidović N, Mestrović S, Dogas Z, et al. Craniofacial morphology of Croatian patients with obstructive sleep apnea. Coll Antropol. 2013;37(1):271-279.

20. Benumof JL. Obstructive sleep apnea in the adult obese patient: implications for airway management. Anesthesiol Clin North America. 2002;20(4):789-811.

21. Perri RA, Kairaitis K, Cistulli P, Weathley JR, Amis TC. Surface cephalometric and anthropometric variables in OSA patients: statistical models for the OSA phenotype. Sleep Breath. 2014;18(1):39-52.

22. Ryan CF, Dickson RI, Lowe AA, Blokmanis A, Fleetham JA. Upper airway measurements predict response to uvulopalatopharyngoplasty in obstructive sleep apnea. Laryngoscope. 1990;100(3):248-253.
23. Barbosa MC, Luégya Amorim HK, LessaIii MM, Martins de Araujo T. Evaluation of lateral cephalometric radiography as a mean of diagnosing adenoids hypertrophy. Dent Press Ortodon Ortop Facial. 2009;14(4):83-91.

24. Souki MQ, Fau SB, Fau FL, Fau BH, Araujo EA. Reliability of subjective, linear, ratio and area cephalometric measurements in assessing adenoid hypertrophy among different age groups. Angle Orthod. 2012;82(6):1001-1007.

25. Koo SK, Choi JW, Myung NS, Lee HJ, Kim YJ. Analysis of obstruction site in obstructive sleep apnea syndrome patients by drug induced sleep endoscopy. Am J Otolaryngol. 2013;34(6):626-630.

26. Shi S, Xia Y, Zhu M, et al. Characterization of upper airway obstruction by fiber-optic nasolaryngoscopy and MRI in preoperative OSAHS patients. ORL J Otorhinolaryngol Relat Spec. 2014;76(6):321-328

27. Ritzel RA, Berwig LC, da Silva AM, Corrêa EC, Serpa EO. Correlation between nasopharyngoscopy and cephalometry in the diagnosis of hyperplasia of the pharyngeal tonsils. Int Arch Otorhinolaryngol. 2012;16(2):209-216.

28. Bates CJ, McDonald JP. The relationship between severity of obstructive sleep apnoea/hypopnoea syndrome (OSAHS) and lateral cephalometric radiograph values: a clinical diagnostic tool. Surgeon. 2005;3(5):338-346.

29. Pahkala R, Seppä J, Ikonen A, Smirnov G, Tuomilehto H. The impact of pharyngeal fat tissue on the pathogenesis of obstructive sleep apnea. Sleep Breath. 2014;18(2):275-282.

30. Abdel-Aziz M, Ibrahim N, Ahmed A, El-Hamamsy M, AbdelKhalik MI, El-Hoshy H. Lingual tonsils hypertrophy; a cause of obstructive sleep apnea in children after adenotonsillectomy: operative problems and management. Int J Pediatr Otorhinolaryngol. 2011;75(9):1127-1131.

31. da Cunha Viana A Jr, Mendes DL, de Andrade Lemes LN, Thuler LC, Neves DD, de Araujo-Melo MH. Drug-induced sleep endoscopy in the obstructive sleep apnea: comparison between NOHL and VOTE classifications. Eur Arch Otorhinolaryngol. 2017;274(2):627-635.

32. Neelapu BC, Kharbanda OP, Sardana HK, et al. Craniofacial and upper airway morphology in adult obstructive sleep apnea patients: a systematic review and meta-analysis of cephalometric studies. Sleep Med Rev. 2017;31:79-90.

33. Genta PR, Schorr F, Eckert DJ, et al. Upper airway collapsibility is associated with obesity and hyoid position. Sleep. 2014;37(10):1673-1678

\section{SUBMISSION \& CORRESPONDENCE INFORMATION}

Submitted for publication June 3, 2017

Submitted in final revised form September 25, 2017

Accepted for publication October 20, 2017

Address correspondence to: Liliana Otero, Carrera 7 No. 40-62, Bogotá, Colombia; Tel: 3208320,x:2899; Email: lotero@javeriana.edu.co

\section{DISCLOSURE STATEMENT}

All authors have seen and approved this manuscript. The authors report no conflicts of interest. This work was funded by COLCIENCIAS through grant 369 Project 501953731808. 